\title{
ELEMENTARY SUBGROUP OF AN ISOTROPIC REDUCTIVE GROUP IS PERFECT
}

\author{
A. YU. LUZGAREV AND A. K. STAVROVA
}

To our Teacher Nikolai Vavilov, on the occasion of his 60th birthday

\begin{abstract}
Let $G$ be an isotropic reductive algebraic group over a commutative ring $R$. Assume that the elementary subgroup $E(R)$ of the group of points $G(R)$ is well defined. Then $E(R)$ is perfect, except for the well-known case of a split reductive group of type $C_{2}$ or $G_{2}$.
\end{abstract}

\section{INTRODUCTION}

Let $R$ be a commutative ring with 1 , and let $G$ be an isotropic reductive algebraic group over $R$. In [1, Victor Petrov and the second author introduced the notion of an elementary subgroup $E(R)$ of the group of points $G(R)$. In this paper we prove that, as in the split case (see, e.g., [11) and in the field case (see, e.g., 12]), under some natural assumptions the elementary subgroup of a reductive group is perfect.

More precisely, assume that $G$ is isotropic in the following strong sense: it possesses a parabolic subgroup that intersects properly any semisimple normal subgroup of $G$. Such a parabolic subgroup $P$ is said to be strictly proper. Let $E_{P}(R)$ denote the subgroup of $G(R)$ generated by the $R$-points of the unipotent radicals of $P$ and of an opposite parabolic subgroup $P^{-}$. The main theorem of [1] states that $E_{P}(R)$ does not depend on the choice of $P$, as soon as for any maximal ideal $M$ of $R$ all irreducible components of the relative root system of $G_{R_{M}}$ (see [9. Exp. XXVI, §7] for the definition) are of rank at least 2. Under this assumption, we call $E_{P}(R)$ the elementary subgroup of $G(R)$ and denote it simply by $E(R)$. In particular, $E(R)$ is normal in $G(R)$. This definition of $E(R)$ generalizes the well-known definition of an elementary subgroup of a Chevalley group (or, more generally, of a split reductive group), as well as several other definitions of an elementary subgroup of isotropic classical groups and simple groups over fields; see [5, 12, 13, 14, 6].

By the structure constants of a root system we mean the structure constants of the corresponding semisimple complex Lie group, or, in other words, the constants appearing in the Chevalley commutator formulas for the corresponding Chevalley group. They are among $\pm 1, \pm 2, \pm 3$.

Theorem 1. Let $G$ be an isotropic reductive algebraic group over a commutative ring $R$. Assume that for any maximal ideal $M$ of $R$ all irreducible components of the relative root

2010 Mathematics Subject Classification. Primary 20 G35.

Key words and phrases. Reductive group, affine group scheme, elementary subgroup, Chevalley commutator formula.

Supported by the research program 6.38.74.2011 "The Structural Theory and Geometry of Algebraic Groups and Their Applications in Representation Theory and Algebraic K-Theory" of St. Petersburg State University and by the RFBR projects 09-01-00878, 09-01-90304, 10-01-00551, 10-01-90016. The first author was also supported by the RFBR project 09-01-00874. The second author was also supported by the grants DFG GI 706/1-2 and DFG SFB/TR 45. 
system of $G_{R_{M}}$ are of rank at least 2 , and that, if one of the irreducible components of the (usual) root system of $G_{R_{M}}$ is of type $B_{2}=C_{2}$ or $G_{2}$, then the residue field $R_{M} / M R_{M}$ is not isomorphic to $\mathbb{F}_{2}$. Then $E(R)=[E(R), E(R)]$.

Observe that the first condition of the theorem ensures that the elementary subgroup $E(R)$ of $G(R)$ is well defined, while the second essentially eliminates the well-known cases where the elementary subgroup of a split reductive group is not perfect. Thus, the result is the strongest possible. It should be noted that if we only assume that the rank of relative root systems of $G_{R_{M}}$ is at most 1, the question as to whether individual subgroups $E_{P}(R)$ are perfect is of separate interest.

The proof of Theorem 1 is based on the notion of relative root subschemes (with respect to a parabolic subgroup) of an isotropic group, as introduced in [1, the generalized Chevalley commutator formula [1, Lemma 9], and localization in a Quillen-Suslin style [3]. To shorten the proof, we also make use of the classification of Tits indices for isotropic reductive groups over local rings, obtained in [10].

\section{§1. An abstract Definition of Relative Roots}

In this section we recall the notion of an (abstract) system of relative roots introduced in 1 and prove a technical lemma.

Let $\Phi$ be a reduced root system in a Euclidean space with a scalar product $(-,-)$. Let $\Pi=\left\{\alpha_{1}, \ldots, \alpha_{l}\right\}$ be a fixed system of simple roots of $\Phi$; if $\Phi$ is irreducible, we assume that the numbering follows Bourbaki $[8$. Let $D$ be the Dynkin diagram of $\Phi$. We identify the nodes of $D$ with the corresponding simple roots in $\Pi$. For a subgroup $\Gamma \subseteq \operatorname{Aut}(D)$ and a $\Gamma$-invariant subset $J \subseteq \Pi$, consider the projection

$$
\pi=\pi_{J, \Gamma}: \mathbb{Z} \Phi \longrightarrow \mathbb{Z} \Phi /\langle\Pi \backslash J ; \alpha-\sigma(\alpha), \alpha \in J, \sigma \in \Gamma\rangle .
$$

The set $\Phi_{J, \Gamma}=\pi(\Phi) \backslash\{0\}$ is called the system of relative roots corresponding to the pair $(J, \Gamma)$. The rank of $\Phi_{J, \Gamma}$ is the rank of $\pi(\mathbb{Z} \Phi)$ as a free Abelian group.

It is clear that any relative root $A \in \Phi_{J, \Gamma}$ can be represented as a unique linear combination of relative roots in $\pi(\Pi)$. We say that $A \in \Phi_{J, \Gamma}$ is a positive (respectively, negative) relative root if it is a nonnegative (respectively, nonpositive) linear combination of elements of $\pi(\Pi)$. The sets of positive and negative relative roots will be denoted by $\Phi_{J, \Gamma}^{+}$and $\Phi_{J, \Gamma}^{-}$, respectively. By the level $\operatorname{lev}(A)$ of a relative root $A$ we mean the sum of coefficients in its decomposition.

Observe that $\Gamma$ acts on the set of irreducible components of the root system $\Phi$. If this action is transitive, the system of relative roots $\Phi_{J, \Gamma}$ is irreducible. Clearly, any system of relative roots $\Phi_{J, \Gamma}$ is a disjoint union of irreducible ones; we call them the irreducible components of $\Phi_{J, \Gamma}$.

We need the following lemma.

Lemma 1. Let $\Phi$ be a root system with a fixed set $\Pi$ of simple roots, let $\Gamma$ be a subgroup of $\operatorname{Aut}(D)$ and $J$ a $\Gamma$-invariant subset of $\Pi$. If a relative root $A \in \Phi_{J, \Gamma}$ lies in an irreducible component of rank at least 2 , then there exist noncollinear $B, C \in \Phi_{J, \Gamma}$ such that $A=B+C$ and all relative roots $i B+j C \in \Phi_{J, \Gamma}, i, j>0,(i, j) \neq(1,1)$, have the same sign as $A$ and satisfy $|\operatorname{lev}(i B+j C)|>|\operatorname{lev}(A)|$.

Proof. We may assume that the root system $\Phi$ is irreducible and that $A$ is a positive relative root, that is, $\pi^{-1}(A) \subseteq \Phi^{+}$.

First, assume that $A=k \pi\left(\alpha_{r}\right)$, where $\alpha_{r} \in \Pi$ is a simple root and $k>0$ is a positive integer. Let $\alpha_{s} \in J$ be a simple root such that the $\Gamma$-orbits of $\alpha_{s}$ and $\alpha_{r}$ are distinct, and $\alpha_{s}$ is at the smallest possible distance from $\alpha_{r}$ on the Dynkin diagram. It is easily seen that for any $\alpha \in \pi^{-1}(A)$ there exists $\beta \in \pi^{-1}\left(\alpha_{s}\right)$ such that $(\alpha, \beta)<0$ and, consequently, 
$\alpha+\beta \in \Phi$. Indeed, since $m_{s}(\alpha)=0$ by definition, we can take for $\beta$ the sum of all simple roots in the Dynkin diagram chain between $\alpha_{s}$ and the nearest simple root appearing in the decomposition of $\alpha$. Now we set $B=\pi(\alpha+\beta)$ and $C=\pi(-\beta)$. Clearly, any root in $\pi^{-1}(i B+j C), i, j>0$, contains the summand $i \alpha_{r}$ in its decomposition; thus, it is a positive root. Then $i B+j C$ is a positive relative root for any $i, j>0$. Moreover, $\operatorname{lev}(i B+j C)=\operatorname{lev}(A)$ if and only if $i=j=1$. Since $\pi(\alpha)=k \pi\left(\alpha_{r}\right)$ and $\pi(-\beta)=-\pi\left(\alpha_{s}\right)$, the roots $B$ and $C$ are noncollinear.

Consider the case where $A \neq k \pi\left(\alpha_{r}\right)$ for any $\alpha_{r} \in J$. For every $\alpha \in \pi^{-1}(A)$, there exists a sequence of simple roots $\beta_{1}, \ldots, \beta_{n} \in \Pi$ such that $\alpha=\beta_{1}+\cdots+\beta_{n}$ and $\beta_{1}+\cdots+\beta_{i} \in \Phi$ for any $1 \leq i \leq n$. Let $i$ be the smallest possible index such that $\beta_{i+1}, \ldots, \beta_{n} \in \Delta$. Then $\beta_{i} \in J$ and $\pi\left(\beta_{1}+\cdots+\beta_{i-1}+\beta_{i}\right)=A$. Set $B=\pi\left(\beta_{1}+\cdots+\beta_{i-1}\right)$ and $C=\pi\left(\beta_{i}\right)$. Since $B$ and $C$ are positive relative roots, we have $\operatorname{lev}(i B+j C)>\operatorname{lev}(A)$ for any $i, j>0$ distinct from $i=j=1$. The relative roots $B$ and $C$ are noncollinear because otherwise we would have had $A=k \pi\left(\beta_{i}\right)$ for some $k>0$.

\section{§2. Isotropic REDUCTIVE GROUPS AND RELATIVE ROOT SUBSCHEMES}

In this section we recall some basic notions pertaining to reductive groups over rings; see [9, 1, 2] for a more detailed exposition.

Let $R$ be a commutative ring with 1 , and let $G$ be a reductive group scheme, or reductive group for short, over $R$ (see [9] for the definition). We denote by $G^{\text {ad }}$ and $G^{\text {sc }}$ the corresponding adjoint and simply connected semisimple groups, respectively.

Any reductive algebraic group $G$ over $R$ is split locally in the fpqc-topology on $\operatorname{Spec} R$. If $G$ is of constant type over $R$ (that is, the root system of $G$ is the same at any point of Spec $R$ ), then $G$ is a twisted form of a split reductive algebraic group $G_{0}$ over $R$, given by a cocycle $\xi \in H_{\text {fpqc }}^{1}\left(R, \operatorname{Aut}\left(G_{0}\right)\right)$. Recall that the connected component of $\operatorname{Aut}\left(G_{0}\right)$ is precisely $G_{0}^{\text {ad }}$. The group $G$ is of inner type if $\xi$ is in the image of the natural map $H_{\text {fpqc }}^{1}\left(R, G_{0}^{\text {ad }}\right) \rightarrow H_{\text {fpqc }}^{1}\left(R, \operatorname{Aut}\left(G_{0}\right)\right)$. One can always find a finite Galois extension $S$ of $R$ such that $G_{S}$ is of inner type. The Galois group $\operatorname{Gal}(S / R)$ acts on the Dynkin diagram of each $G_{\overline{k(s)}}$, where $\overline{k(s)}$ is the algebraic closure of the residue field of a point $s \in \operatorname{Spec} R$, via a $*$-action (see [10, 2]).

Recall that $G$ is said to be isotropic if it contains a proper parabolic subgroup $P$ over $R$. Recall that a parabolic subgroup $P$ of $G$ is strictly proper if it does not contain any semisimple normal subgroup of $G$. We set

$$
E_{P}(R)=\left\langle U_{P}(R), U_{P^{-}}(R)\right\rangle,
$$

where $P^{-}$is any parabolic subgroup of $G$ opposite to $P$, and $U_{P}$ and $U_{P^{-}}$are the unipotent radicals of $P$ and $P^{-}$, respectively. The main theorem of [1] states that $E_{P}(R)$ does not depend on the choice of a strictly proper parabolic subgroup $P$, as soon as for any maximal ideal $M$ in $R$ all irreducible components of the relative root system of $G_{R_{M}}$ are of rank at least 2. Under this assumption, we call $E_{P}(R)$ the elementary subgroup of $G(R)$ and denote it simply by $E(R)$.

Let $P=P^{+}$be a parabolic subgroup of $G$ and $P^{-}$an opposite parabolic subgroup. Let $L=P^{+} \cap P^{-}$be their common Levi subgroup. It was shown in [1] that we can represent $\operatorname{Spec}(R)$ as a finite disjoint union

$$
\operatorname{Spec}(R)=\coprod_{i=1}^{m} \operatorname{Spec}\left(R_{i}\right)
$$

so that the following conditions hold true for $i=1, \ldots, m$ :

- for any $s \in \operatorname{Spec} R_{i}$, the root system of $G_{\overline{k(s)}}$ is the same;

- for any $s \in \operatorname{Spec} R_{i}$, the type of the parabolic subgroup $P_{\overline{k(s)}}$ of $G_{\overline{k(s)}}$ is the same; 
- if $S_{i} / R_{i}$ is a Galois extension of rings such that $G_{S_{i}}$ is of inner type, then for any $s \in \operatorname{Spec} R_{i}$ the Galois group $\operatorname{Gal}\left(S_{i} / R_{i}\right)$ acts on the Dynkin diagram $D_{i}$ of $G_{\overline{k(s)}}$ via the same subgroup of $\operatorname{Aut}\left(D_{i}\right)$.

From here until the end of this section, we assume that $R=R_{i}$ for some $i$ (or simply extend the base). Denote by $\Phi$ the root system of $G$, by $\Pi$ a set of simple roots of $\Phi$, and by $D$ the corresponding Dynkin diagram. Then the $*$-action on $D$ is determined by a subgroup $\Gamma$ of Aut $D$. Let $J$ be the subset of $\Pi$ such that $\Pi \backslash J$ is the type of $P \overline{k(s)}$ (that is, the set of simple roots of the Levi subgroup $L \overline{k(s)})$. Then $J$ is $\Gamma$-invariant. The system of relative roots $\Phi_{J, \Gamma}$ is called the system of relative roots corresponding to $P$ and is denoted also by $\Phi_{P}$. If $R$ is a local ring and $P$ is a minimal parabolic subgroup of $G$, then $\Phi_{P}$ can be identified with the relative root system of $G$ in the sense of [9. Exp. XXVI, §7] (or [7] for the field case); see also [7, 1, 2].

With any relative root $A \in \Phi_{P}$, we associate a finitely generated projective $R$-module $V_{A}$ and a closed embedding

$$
X_{A}: W\left(V_{A}\right) \rightarrow G,
$$

where $W\left(V_{A}\right)$ is the affine group scheme over $R$ defined by $V_{A}$ and called a relative root subscheme of $G$. These subschemes possess several nice properties similar to those of elementary root subgroups of a split group; see [1, Theorem 2]. In particular, they are subject to certain commutator relations that generalize the Chevalley commutator formula.

More precisely, assume that $A, B \in \Phi_{P}$ satisfy $m A \neq-k B$ for any $m, k \geq 1$. Then there exists a polynomial map

$$
N_{A B i j}: V_{A} \times V_{B} \rightarrow V_{i A+j B}
$$

homogeneous of degree $i$ in the first variable and of degree $j$ in the second variable and such that for any $R$-algebra $R^{\prime}$ and any $u \in V_{A} \otimes_{R} R^{\prime}, v \in V_{B} \otimes_{R} R^{\prime}$ we have

$$
\left[X_{A}(u), X_{B}(v)\right]=\prod_{i, j>0} X_{i A+j B}\left(N_{A B i j}(u, v)\right)
$$

(see [1, Lemma 9]).

In a strict analogy with the split case, for any $R$-algebra $R^{\prime}$ we have

$$
E\left(R^{\prime}\right)=\left\langle X_{A}\left(V_{A} \otimes_{R} R^{\prime}\right), A \in \Phi_{P}\right\rangle
$$

(see [1, Lemma 6]).

We shall also use the following statement, which is a slight extension of [1, Lemma 10].

Lemma 2. Consider $A, B \in \Phi_{P}$ satisfying $A+B \in \Phi_{P}$ and $m A \neq-k B$ for any $m, k \geq 1$. Denote by $\Phi^{0}$ an irreducible component of $\Phi$ such that $A, B \in \pi\left(\Phi^{0}\right)$.

(1) Suppose that one of the following conditions is fulfilled:

(a) the structure constants of $\Phi^{0}$ are invertible in $R$ (for example, this is so if $\Phi^{0}$ is simply laced);

(b) $A \neq B$ and $A-B \notin \Phi_{P}$;

(c) $\Phi^{0}$ is of type $B_{l}, C_{l}$, or $F_{4}$, and $\pi^{-1}(A+B)$ consists of short roots;

(d) $\Phi^{0}$ is of type $B_{l}, C_{l}$, or $F_{4}$, and there exist long roots $\alpha \in \pi^{-1}(A), \beta \in \pi^{-1}(B)$ such that $\alpha+\beta$ is a root.

Then the map $N_{A B 11}: V_{A} \times V_{B} \rightarrow V_{A+B}$ is surjective.

(2) If $A-B \in \Phi_{P}$ and $\Phi^{0} \neq G_{2}$, then

$$
\operatorname{im} N_{A B 11}+\operatorname{im} N_{A-B, 2 B, 1,1}+\sum_{v \in V_{B}} \operatorname{im}\left(N_{A-B, B, 1,2}(-, v)\right)=V_{A+B},
$$

where $\operatorname{im} N_{A-B, 2 B, 1,1}=0$ if $2 B \notin \Phi_{P}$. 
Proof. (1) By [1, Lemma 4], any $\gamma \in \pi^{-1}(A+B)$ decomposes as $\gamma=\alpha+\beta, \alpha \in \pi^{-1}(A)$, $\beta \in \pi^{-1}(B)$. Let $S_{\tau}$ be any member of an affine fpqc-covering $\amalg \operatorname{Spec} S_{\tau} \rightarrow \operatorname{Spec} R$ that splits $G$. Set

$$
\Psi=\left\{i A+j B \mid i, j>0,(i, j) \neq(1,1), i A+j B \in \Phi_{P}\right\} .
$$

Then, in the notation of [1, Theorem 2], over $S_{\tau}$ the commutator $\left[X_{A}\left(e_{\alpha}\right), X_{B}\left(e_{\beta}\right)\right]$ computed modulo the subgroup $\left\langle X_{C}\left(V_{C}\right), C \in \Psi\right\rangle$, is of the form $x_{\gamma}( \pm c)=X_{A+B}\left( \pm c e_{\gamma}\right)$, where $c= \pm 1, \pm 2, \pm 3$ is the corresponding structure constant. If (a) is fulfilled, then $c$ is invertible. If (b), (c), or (d) hold true, then $c$ necessarily equals \pm 1 . Indeed, in the only dubious case (d) we should observe that, due to the transitive action of the Weyl group of the Levi subgroup on the roots of the same shape (see [4]), any long root $\gamma \in \pi^{-1}(A+B)$ decomposes as a sum of long roots. Hence, $c$ is always invertible. This implies that $\operatorname{im}\left(N_{A B 11}\right)_{\tau}=V_{A+B} \otimes S_{\tau}$. Since im $N_{A B 11}$ is a submodule of $V_{A+B}$ defined over the base ring, we have $\operatorname{im} N_{A B 11}=V_{A+B}$.

(2) See [1, Lemma 10].

Lemma 3. Suppose that $\Phi^{0}$ is an irreducible component of $\Phi$ such that $\Phi^{0} \cong C_{l}, l>2$, and $P$ is a parabolic subgroup of type $\Pi \backslash J$, where $J \cap \Phi^{0}=\left\{\alpha_{i}, \alpha_{l}\right\}, 2 i=l$ ( $\alpha_{i}$ is short, $\alpha_{l}$ is long $)$. Set $\pi\left(\alpha_{i}\right)=A_{1}$ and $\pi\left(\alpha_{l}\right)=A_{2}$. Then

$$
\operatorname{im}\left(0, N_{A_{1}, A_{1}+A_{2}, 1,1}\right)+\sum_{v \in V_{A}} \operatorname{im} f_{v}=V_{A_{1}+A_{2}} \oplus V_{2 A_{1}+A_{2}},
$$

where $f_{v}=\left(N_{A_{1}, A_{2}, 1,1}(v,-), N_{A_{1}, A_{2}, 2,1}(v,-)\right): V_{A_{2}} \rightarrow V_{A_{1}+A_{2}} \oplus V_{2 A_{1}+A_{2}}$.

Proof. Observe that in our case $\pi\left(\Phi^{0}\right)$ can be identified with a root system of type $C_{2}$, so that $A_{1}$ is a short simple root and $A_{2}$ is a long simple root. Let $S_{\tau}$ be any member of an affine fpqc-covering $\coprod \operatorname{Spec} S_{\tau} \rightarrow \operatorname{Spec} R$ that splits $G$. Suppose that $\gamma \in \pi^{-1}\left(2 A_{1}+A_{2}\right)$ is a short root. We can find short roots $\alpha \in \pi^{-1}\left(A_{1}\right)$ and $\beta \in \pi^{-1}\left(A_{1}+A_{2}\right)$ such that $\gamma=\alpha+\beta$. Therefore,

$$
\left[X_{A_{1}}\left(e_{\alpha}\right), X_{A_{1}+A_{2}}\left(e_{\beta}\right)\right]=x_{\gamma}( \pm 1)=X_{2 A_{1}+A_{2}}\left( \pm e_{\gamma}\right) .
$$

Hence, $e_{\gamma} \in \operatorname{im}\left(N_{A_{1}, A_{1}+A_{2}, 1,1}\right)_{\tau}$. Now let $\gamma \in \pi^{-1}\left(2 A_{1}+A_{2}\right)$ be a long root. Take $\alpha \in \pi^{-1}\left(A_{1}\right), \beta \in \pi^{-1}\left(A_{2}\right)$ such that $\beta \neq \alpha_{l}$ and $\gamma=2 \alpha+\beta$ (note that $\alpha$ is short, $\beta$ is long). Then

$$
\left[X_{A_{1}}\left(e_{\alpha}\right), X_{A_{2}}\left(e_{\beta}\right)\right]=x_{\alpha+\beta}( \pm 1) x_{2 \alpha+\beta}( \pm 1)=X_{A_{1}+A_{2}}\left( \pm e_{\alpha+\beta}\right) X_{2 A_{1}+A_{2}}\left( \pm e_{\gamma}\right) .
$$

Next, since any $\delta \in \pi^{-1}\left(A_{1}+A_{2}\right)$ is a short root, there exist short roots $\alpha \in \pi^{-1}\left(A_{1}\right), \beta \in$ $\pi^{-1}\left(A_{2}\right)$ such that $\alpha+\beta=\delta$, whence $\left[X_{A_{1}}\left(e_{\alpha}\right), X_{A_{2}}\left(e_{\beta}\right)\right]=X_{A_{1}+A_{2}}\left( \pm e_{\delta}\right)$. Combining these results, we see that (2) is valid over $S_{\tau}$. Since all our modules are defined over the base ring, fpqc descent shows that the same identity is valid over $R$.

\section{§3. Proof of Theorem 1}

Let $R$ be a commutative ring with $1, G$ a reductive group over $R$, and $P$ a strictly proper parabolic subgroup of $G$. For any ideal $I \subseteq R$ we write

$$
E_{P}(I)=\left\langle U_{P}(I), U_{P^{-}}(I)\right\rangle \leq G(R) .
$$

We denote by $R[Y, Z]$ the ring of polynomials in two variables $Z$ and $Y$ over $R$.

Lemma 4. Let $G$ be a reductive group scheme over a commutative ring $R$, and let $P$ and $P^{\prime}$ be two strictly proper parabolic subgroups of $G$ such that $P \leq P^{\prime}$ or $P^{\prime} \leq P$. Then for any integer $m>0$ there exists an integer $k>0$ such that

$$
E_{P}\left(Z^{k} R[Z]\right) \leq E_{P^{\prime}}\left(Z^{m} R[Z]\right) .
$$


Proof. Without loss of generality, we may assume that over $R$ we have two sets of relative root subschemes $X_{A}\left(V_{A}\right), A \in \Phi_{P}$, and $X_{B}\left(V_{B}\right), B \in \Phi_{P^{\prime}}$, corresponding to $P$ and $P^{\prime}$, respectively.

Then, if $P \leq P^{\prime}$, by [1, Lemma 12] there exists an integer $k>0$ such that for any $A \in \Phi_{P}$ and any $v \in V_{A}$ we can find relative roots $B_{i} \in \Phi_{P^{\prime}}$, elements $v_{i} \in V_{B_{i}}$, and integers $n_{i}>0(1 \leq i \leq m)$ such that

$$
X_{A}\left(Y^{k} v\right)=\prod_{i=1}^{m} X_{B_{i}}\left(Y^{n_{i}} v_{i}\right)
$$

so that $X_{A}\left(Y^{k} v\right) \in E_{P^{\prime}}(Y R[Y])$. Replacing $R$ by $R[Z]$ and $Y$ by $Z^{m}$, we obtain

$$
E_{P}\left(Z^{k} R[Z]\right) \leq E_{P^{\prime}}\left(Z^{m} R[Z]\right) .
$$

But if $P^{\prime} \leq P$, we have $U_{P} \leq U_{P^{\prime}}$. Let $\Psi^{ \pm} \subseteq \Phi^{+}$be two closed sets of roots corresponding to $U_{P^{ \pm}}$. Then $\pi\left(\Psi^{ \pm}\right) \subseteq \Phi_{P^{\prime}}^{ \pm}$, where $\pi: \Phi \rightarrow \Phi_{P^{\prime}}$ is the canonical projection. By [1, Lemma 6], the map

$$
X_{\Psi}: \mathrm{W}\left(\bigoplus_{A \in \pi\left(\Psi^{ \pm}\right)} V_{A}\right) \rightarrow \mathrm{U}_{P^{ \pm}}, \quad\left(v_{A}\right)_{A} \mapsto \prod_{A} X_{A}\left(v_{A}\right),
$$

where the product is taken in any fixed order respecting the level of relative roots in $\Phi_{P^{\prime}}$, is an isomorphism of schemes over $R$. Therefore, $U_{P^{ \pm}}\left(Z^{k} R[Z]\right) \leq U_{P^{\prime \pm}}\left(Z^{m} R[Z]\right)$.

Lemma 5. Under the conditions of Theorem 1, assume moreover that $R$ is a local ring. Then for any integer $m>0$ there exists an integer $k>0$ such that for any $R$-algebra $R^{\prime}$ we have

$$
E_{P}\left(Z^{k} R^{\prime}[Z]\right) \subseteq\left[E_{P}\left(Z^{m} R^{\prime}[Z]\right), E_{P}\left(Z^{m} R^{\prime}[Z]\right)\right] .
$$

Proof. Let $\operatorname{der}(G)$ be the algebraic derived subgroup of the reductive group scheme $G$ (see [9, Exp. XXII, 6.2]). Then, clearly, $E_{P}(R) \subseteq \operatorname{der}(G)(R)$. Since $\operatorname{der}(G)$ is a semisimple group, we may assume that $G$ is semisimple. Moreover, since the canonical projection $G^{s c} \rightarrow G$, where $G^{s c}$ is the simply connected semisimple group corresponding to $G$, is surjective on $U^{ \pm}(R)$, we may assume that $G$ is simply connected. Any simply connected semisimple group is a direct product of simply connected semisimple groups that cannot be decomposed into a product of smaller semisimple groups. These groups $G_{i}$, $i=1, \ldots, n$, are Weil restrictions of certain simple reductive groups $G_{i}^{\prime}$ over a finite étale extension $S$ of $R: G_{i}=\mathrm{R}_{S / R}\left(G_{i}^{\prime}\right)$; see [9, Exp. XIV, Proposition 5.10].

Note that the group of $R$-points $G_{i}(R)$ is canonically isomorphic to the group of $S$-points $G_{i}^{\prime}(S)$. This isomorphism also respects the embedding $P_{i}(R) \rightarrow G_{i}(R)$, for any parabolic subgroup $P_{i}$ of $G_{i}$. Then, clearly, we may assume from the very beginning that $G$ is a simple reductive group, and the root system $\Phi$ of $G$ is irreducible.

Note that Lemma 4 allows us to replace $P$ by any other strictly proper parabolic subgroup $P^{\prime}$ of $G$ such that $P \leq P^{\prime}$ or $P^{\prime} \leq P$. Next, over $R$ we have a set of relative root subschemes $X_{A}\left(V_{A}\right), A \in \Phi_{P}$, corresponding to $P$.

We are going to show by induction on $|\operatorname{lev} A|$ that for any $A \in \Phi_{P}$ there exists an integer $k=k(A)>0$ such that for any $R$-algebra $R^{\prime}$ and any $v \in V_{A} \otimes_{R} R^{\prime}$ we have

$$
X_{A}\left(Z^{k} v\right) \in\left[E_{P}\left(Z R^{\prime}[Z]\right), E_{P}\left(Z R^{\prime}[Z]\right)\right] .
$$

Then the claim of the lemma follows by replacing $Z$ by $Z^{m}$ and $R^{\prime}$ by $R[Z]$, and taking the final $k$ to be the maximum of all $k(A), A \in \Phi_{P}$.

Recall that, by Lemma 1, there exist noncollinear relative roots $B, C \in \Phi_{P}$ such that $A=B+C$ and all roots $i B+j C \in \Phi_{J, \Gamma}, i, j>0,(i, j) \neq(1,1)$, have the same sign as $A$ and satisfy $|\operatorname{lev}(i B+j C)|>|\operatorname{lev}(A)|$. Assume that the map $N_{B C 11}: V_{B} \times V_{C} \rightarrow V_{A}$ is 
surjective. Using the generalized Chevalley commutator formula (1), for any $R$-algebra $R^{\prime}$ and any $v \in V_{A} \otimes_{R} R^{\prime}[Z]$ we can write

$$
X_{A}\left(Z^{k} v\right)=\left[X_{B}\left(Z u_{10}\right), X_{C}\left(Z^{k-1} u_{01}\right)\right] \cdot \prod_{\substack{i, j>0 ; \\(i, j) \neq(1,1)}} X_{i B+j C}\left(Z^{i+j(k-1)} u_{i j}\right)
$$

for some $u_{i j} \in V_{i B+j C} \otimes_{R} R^{\prime}[Z], i, j>0,(i, j) \neq(1,1)$. Then, by the inductive hypothesis, (3) is true if $k$ is sufficiently large.

Now, for any relative root $A \in \Phi_{P}$ (for a suitable choice of the parabolic subgroup $P$ ), we either show that for any decomposition $A=B+C$, where $B$ and $C$ are noncollinear, the map $N_{B C 11}$ is surjective; or provide an explicit decomposition of $X_{A}\left(Z^{k} v\right)$, $v \in V_{A} \otimes_{R} R^{\prime}$, into a product of commutators in $E_{P}\left(Z R^{\prime}[Z]\right)$, so that (3) is satisfied for sufficiently large $k$.

First, assume that all structure constants of the root system $\Phi$ of $G$ are invertible in $R$; this includes the case where $\Phi$ is simply laced. Then, by Lemma 2 the map $N_{B C 11}$ is surjective for any decomposition $A=B+C$, where $B$ and $C$ are noncollinear.

Consider the case where $\Phi=\Phi_{P}=C_{2}$, so that $G$ is split. Let $\Pi=\left\{A_{1}, A_{2}\right\}$, $\Phi^{+}=\left\{A_{1}, A_{2}, A_{1}+A_{2}, 2 A_{1}+A_{2}\right\}$. Let $M$ be the maximal ideal of $R$. By the hypotheses of Theorem 1. we have $R / M \neq \mathbb{F}_{2}$; hence, we can take $\varepsilon \in R \backslash M$ such that $\varepsilon^{2}-\varepsilon \in$ $R \backslash M=R^{*}$. If the root $A \in \Phi_{P}$ is long, we may assume that $A=2 A_{1}+A_{2}$. Let

$$
g_{1}(s, t)=\left[X_{A_{1}}(s), X_{A_{2}}(t)\right]=X_{A_{1}+A_{2}}(s t) X_{2 A_{1}+A_{2}}\left(s^{2} t\right)
$$

and

$$
g_{2}(s, t, u)=\left[X_{A_{2}}(u),\left[X_{A_{1}+A_{2}}(s), X_{-A_{2}}(t)\right]\right]=X_{A_{1}+A_{2}}(-s t u) X_{2 A_{1}+A_{2}}\left(-s^{2} t^{2} u\right) .
$$

Here and in similar calculations below, we use the explicit signs of the structure constants in a split group; see, e.g., [11. Therefore,

$$
g_{1}\left(Z^{2},-Z^{k-4} \varepsilon\left(\varepsilon^{2}-\varepsilon\right)^{-1} v\right) \cdot g_{2}\left(Z, Z \varepsilon,-Z^{k-4}\left(\varepsilon^{2}-\varepsilon\right)^{-1} v\right)=X_{2 A_{1}+A_{2}}\left(Z^{k} v\right) .
$$

But if the root $A \in \Phi_{P}$ is short, we may assume that $A=A_{1}+A_{2}$, whence

$$
g_{1}\left(Z, Z^{k-1} v\right) \cdot X_{2 A_{1}+A_{2}}\left(-Z^{k+1} v\right)=X_{A_{1}+A_{2}}\left(Z^{k} v\right) \text {. }
$$

This means that (3) holds true for these roots for any $k \geq 5$.

Consider the case where $\Phi=\Phi_{P}=G_{2}$, so that $G$ is split. Let $\Pi=\left\{A_{1}, A_{2}\right\}$, $\Phi^{+}=\left\{A_{1}, A_{2}, A_{1}+A_{2}, 2 A_{1}+A_{2}, 3 A_{1}+A_{2}, 3 A_{1}+2 A_{2}\right\}$. By the hypotheses of Theorem 1 , we have $R / M \nsubseteq \mathbb{F}_{2}$; hence, we can take $\varepsilon \in R \backslash M$ such that $\varepsilon^{2}-\varepsilon \in R \backslash M=R^{*}$. If the root $A$ is long, we may assume that $A=3 A_{1}+2 A_{2}$. Then

$$
\left[X_{A_{2}}(Z v), X_{3 A_{1}+A_{2}}\left(Z^{k-1}\right)\right]=X_{3 A_{1}+2 A_{2}}\left(Z^{k} v\right) \text {. }
$$

Therefore, (3) is valid for long roots for any $k \geq 2$. If the root $A$ is short, we may assume that $A=2 A_{1}+A_{2}$. Then

$$
\left[X_{A_{1}}(s), X_{A_{2}}(t)\right]=X_{A_{1}+A_{2}}(s t) \cdot X_{2 A_{1}+A_{2}}\left(s^{2} t\right) \cdot X_{3 A_{1}+A_{2}}\left(s^{3} t\right) \cdot X_{3 A_{1}+2 A_{2}}\left(s^{3} t^{2}\right) .
$$

Hence,

$$
\begin{aligned}
& {\left[X_{A_{1}}(Z \varepsilon), X_{A_{2}}\left(-\left(\varepsilon^{2}-\varepsilon\right)^{-1} Z^{k-2} v\right)\right]^{-1} \cdot\left[X_{A_{1}}(Z), X_{A_{2}}\left(-\varepsilon\left(\varepsilon^{2}-\varepsilon\right)^{-1} Z^{k-2} v\right)\right]} \\
& \quad=X_{2 A_{1}+A_{2}}\left(Z^{k} v\right) X_{3 A_{1}+A_{2}}\left((\varepsilon+1) Z^{k+1} v\right) X_{3 A_{1}+2 A_{2}}\left(\varepsilon\left(\varepsilon^{2}-\varepsilon\right)^{-1} Z^{2 k+1} v\right)
\end{aligned}
$$

and the roots $3 A_{1}+A_{2}$ and $3 A_{1}+2 A_{2}$ are long. This means that (3) is fulfilled for short roots for any $k \geq 3$.

We are left with the case where $\Phi$ is of type $B_{l}, C_{l}$, or $F_{4}$. Recall that, by the hypotheses of Theorem 1, $G$ contains a split torus of rank at least 2. Hence, in the $F_{4}$ case the classification of Tits indices over local rings [10] says that $G$ is a split group. 
Thus, we may assume that $P$ is a Borel subgroup of $G$, and $\Phi_{P}=\Phi$ is a root system of type $F_{4}$. Then if the root $A \in \Phi_{P}$ is short, Lemma 2 shows that the map $N_{B C 11}$ is surjective for any noncollinear $B, C \in \Phi_{P}$ such that $A=B+C$. If this root is long, then it belongs to the long root subsystem of $F_{4}$, which has type $D_{4}$. Then (for example, by Lemma 10 we can find two long roots $B, C \in \Phi_{P}$ such that $B+C=A$, and necessarily, $i B+j C$ is not a root for any $i, j>0$ distinct from $i=j=1$. Then

$$
X_{A}\left(Z^{k} v\right)=\left[X_{B}\left(Z u_{10}\right), X_{C}\left(Z^{k-1} u_{01}\right)\right]
$$

for some $u_{10} \in V_{B} \otimes_{R} R^{\prime}[Z]$ and $u_{01} \in V_{C} \otimes_{R} R^{\prime}[Z]$.

Consider the case where $\Phi$ is of type $B_{l}, l \geq 3$. By the classification of Tits indices over local rings [10], we may assume that $P$ is a parabolic subgroup of type $\Pi \backslash J$, where $\Pi$ is a set of simple roots of $\Phi$ and $J=\left\{\alpha_{1}, \alpha_{2}\right\}$. Then $\Phi_{P}$ can be identified with a root system of type $B_{2}$. Using the fact that $l \geq 3$, it is easy to check that for any relative root $A \in \Phi_{P}$ and any pair $B, C \in \Phi_{P}$ satisfying $A=B+C$, we can find a pair of long roots $\beta \in \pi^{-1}(B), \gamma \in \pi^{-1}(C)$ such that $\beta+\gamma$ is a root (we may assume that $A$ is one of two simple roots of $\Phi_{P}$, due to the lifting of the relative Weyl group 9, Exp. XXVI, Theorem 7.13 (ii)]). Then, the map $N_{B C 11}$ is surjective by Lemma 2 .

It remains to consider the case where $\Phi$ is of type $C_{l}, l \geq 3$. First, assume that $P$ is a parabolic subgroup of type $\Pi \backslash J$, where $J=\left\{\alpha_{i}, \alpha_{j}\right\}$ for two short simple roots $\alpha_{i}, \alpha_{j}$ of $\Phi$. Then $\Phi_{P}$ can be identified with a root system of type $B C_{2}$. It is easily seen that for all extrashort and short relative roots $A \in \Phi_{P}$ the set $\pi^{-1}(A)$ consists of short roots, and hence, by Lemma 2, the map $N_{B C 11}$ is surjective for any decomposition $A=B+C$. Let $A$ be a long root. Let $A_{1}$ and $A_{2}$ be a short and an extrashort simple roots of $\Phi_{P}$. There is no loss of generality in assuming that $A=2 A_{1}+2 A_{2}$. Take $k \geq 4$. Then, by Lemma 2 (2) and the generalized Chevalley commutator formula, for any $R$-algebra $R^{\prime}$ and any $v \in V_{A} \otimes_{R} R^{\prime}[Z]$ we have

$$
X_{A}\left(Z^{k} v\right)=\left[X_{A_{1}}\left(Z u_{1}\right), X_{2 A_{2}}\left(Z^{k-2} u_{2}\right)\right] \cdot X_{A_{1}+2 A_{2}}\left(Z^{k-1} u_{3}\right)
$$

for some $u_{1} \in V_{A_{1}} \otimes_{R} R^{\prime}[Z], u_{2} \in V_{2 A_{2}} \otimes_{R} R^{\prime}[Z]$, and $u_{3} \in V_{A_{1}+2 A_{2}} \otimes_{R} R^{\prime}[Z]$. Next, by Lemma 2 (1) and the generalized Chevalley commutator formula, since $\pi^{-1}\left(A_{1}+2 A_{2}\right)$ consists of short roots, we have

$$
X_{A_{1}+2 A_{2}}\left(Z^{k-1} u_{3}\right)=\left[X_{A_{1}+A_{2}}\left(Z u_{4}\right), X_{A_{2}}\left(Z^{k-3} u_{5}\right)\right]
$$

for some $u_{4} \in V_{A_{1}+A_{2}} \otimes_{R} R^{\prime}[Z]$ and $u_{5} \in V_{A_{2}} \otimes_{R} R^{\prime}[Z]$. Consequently, (3) holds true for $A$ for any $k \geq 4$.

The classification of Tits indices over local rings [10] shows that the only remaining case is that where $P$ is a parabolic subgroup of type $\Pi \backslash J$ for $J=\left\{\alpha_{i}, \alpha_{l}\right\}$, where $l=2 i$. Now $\alpha_{i}$ is short, $\alpha_{l}$ is long, and $\Phi_{P}$ can be identified with a root system of type $B_{2}=C_{2}$. As in Lemma 3, we put $A_{1}=\pi\left(\alpha_{i}\right), A_{2}=\pi\left(\alpha_{l}\right)$. Then if the root $A \in \Phi_{P}$ is short, by the lifting of the relative Weyl group [9, Exp. XXVI, Theorem 7.13 (ii)]), we may assume that $A=A_{1}+A_{2}$. By Lemma 3, for any $R$-algebra $R^{\prime}$ and any $v \in V_{A} \otimes_{R} R^{\prime}[Z]$, we have

$$
X_{A}\left(Z^{k} v\right)=\prod_{i}\left[X_{A_{1}}\left(Z v_{i}\right), X_{A_{2}}\left(Z^{k-1} u_{1}\right)\right]
$$

for some $u_{1} \in V_{A_{2}} \otimes_{R} R^{\prime}[Z], v_{i} \in V_{A_{1}} \otimes_{R} R^{\prime}[Z]$. If the root $A \in \Phi_{P}$ is long, we may assume that $C=2 A_{1}+A_{2}$. By Lemma 3, for any $R$-algebra $R^{\prime}$ and any $v \in V_{2 A_{1}+A_{2}} \otimes_{R} R^{\prime}[Z]$, we have

$$
X_{A}\left(Z^{k} v\right)=\left[X_{A_{1}}\left(Z u_{1}\right), X_{A_{1}+A_{2}}\left(Z^{k-1} u_{2}\right)\right] \cdot \prod_{i}\left[X_{A_{1}}\left(Z v_{i}\right), X_{A_{2}}\left(Z^{k-2} u_{3}\right)\right]
$$

for some $u_{1} \in V_{A_{1}} \otimes_{R} R^{\prime}[Z], u_{2} \in V_{A_{1}+A_{2}} \otimes_{R} R^{\prime}[Z], u_{3} \in V_{A_{2}} \otimes_{R} R^{\prime}[Z]$, and $v_{i} \in$ $V_{A_{1}} \otimes_{R} R^{\prime}[Z]$. Hence, (3) is true for $A$ for any $k \geq 3$. 
Proof of Theorem 1, Recall that we can represent $\operatorname{Spec}(R)$ as a finite disjoint union $\operatorname{Spec}(R)=\coprod_{i=1}^{n} \operatorname{Spec}\left(R_{i}\right)$, so that $E(R)=\prod_{i=1}^{n} E\left(R_{i}\right)$ and for any $1 \leq i \leq n$ we have

$$
E\left(R_{i}\right)=\left\langle X_{A}\left(V_{A}\right), A \in \Phi_{P_{R_{i}}}\right\rangle
$$

for a set of root subschemes $X_{A}, A \in \Phi_{P_{R_{i}}}$, over $R_{i}$. Hence, we may assume that

$$
E(R)=\left\langle X_{A}\left(V_{A}\right), A \in \Phi_{P}\right\rangle
$$

from the very beginning.

We show that $[E(R), E(R)]$ contains any $X_{A}(v), A \in \Phi_{P}, v \in V_{A}$, by induction on | lev $A \mid$. Take

$$
I=\left\{s \in R \mid X_{A}(t s v) \in[E(R), E(R)] \text { for all } t \in R\right\} .
$$

By [1, Theorem 2], for any $u, u^{\prime} \in V_{A}$ we have

$$
X_{A}\left(u+u^{\prime}\right)=X_{A}(u) X_{A}\left(u^{\prime}\right) \prod_{i>0} X_{i A}\left(u_{i}\right)
$$

for some $u_{i} \in V_{i A}$. Hence, by the inductive hypothesis, $I$ is an ideal of $R$. If $I \neq R$, let $M$ be a maximal ideal of $R$ containing $I$. Let $F_{M}$ denote both the localization homomorphism $R \rightarrow R_{M}$ and the induced homomorphism $G(R[Y, Z]) \rightarrow G\left(R_{M}[Y, Z]\right)$. By Lemma 5 there is $m>0$ such that we can represent the element $F_{M}\left(X_{A}\left(Z^{m} Y v\right)\right)$ of $G\left(R_{M}[Y, Z]\right)$ as a product

$$
F_{M}\left(X_{A}\left(Z^{m} Y v\right)\right)=\prod_{i=1}^{n}\left[h_{i}(Y, Z), g_{i}(Y, Z)\right]
$$

for some $h_{i}(Y, Z), g_{i}(Y, Z) \in E\left(Z R_{M}[Y, Z]\right), 1 \leq i \leq n$. By [1, Lemma 15], there exist

$$
h_{i}^{\prime}(Y, Z), g_{i}^{\prime}(Y, Z) \in E_{P}(R[Y, Z], Z R[Y, Z]) \leq E_{P}(R[Y, Z])=E(R[Y, Z])
$$

and $s \in R \backslash M$ such that $F_{M}\left(h_{i}^{\prime}(Y, Z)\right)=h_{i}(Y, s Z)$ and $F_{M}\left(g_{i}^{\prime}(Y, Z)\right)=g_{i}(Y, s Z)$ for all $i$. Consequently,

$$
F_{M}\left(X_{A}\left((s Z)^{m} Y v\right)\right)=F_{M}\left(\prod_{i=1}^{n}\left[h_{i}^{\prime}(Y, Z), g_{i}^{\prime}(Y, Z)\right]\right) .
$$

Using [1, Lemma 14], we see that there exists $t \in R \backslash M$ with

$$
X_{A}\left((t s Z)^{m} Y v\right)=\prod_{i=1}^{n}\left[h_{i}^{\prime}(Y, t Z), g_{i}^{\prime}(Y, t Z)\right] .
$$

Replacing $Z$ by 1 and $Y$ by an arbitrary element of $R$, we conclude that $(t s)^{m} \in I$. But $(t s)^{m} \in R \backslash M$, a contradiction.

The authors are sincerely grateful to Victor Petrov and Nikolai Vavilov for their inspiring comments on the subject of this paper.

\section{REFERENCES}

[1] V. A. Petrov and A. K. Stavrova, Elementary subgroups of isotropic reductive groups, Algebra i Analiz 20 (2008), no. 4, 160-188; English transl., St. Petersburg Math. J. 20 (2009), no. 4, 625-644. MR.2473747(2009j:20069)

[2] A. K. Stavrova, The structure of isotropic reductive groups, Kand. diss., S.-Peterburg. Univ., St. Petersburg, 2009. (Russian)

[3] A. A. Suslin, The structure of the special linear group over rings of polynomials, Izv. Akad. Nauk SSSR Ser. Mat. 41 (1977), no. 2, 235-252; English transl. in Math. USSR-Izv. 11 (1977), no. 1. $\operatorname{MR} 0472792(57: 12482)$

[4] H. Azad, M. Barry, and G. Seitz, On the structure of parabolic subgroups, Comm. Algebra 18 (1990), 551-562. MR1047327(91d:20048) 
[5] H. Bass, K-theory and stable algebra, Inst. Hautes Études Sci. Publ. Math. No. 22 (1964), 5-60. MR0174604 (30:4805)

[6] A. Bak and N. Vavilov, Structure of hyperbolic unitary groups. I. Elementary subgroups, Algebra Colloq. 7 (2000), 159-196. MR1810843 (2002b:20070)

[7] A. Borel and J. Tits, Groupes réductifs, Inst. Hautes Études Sci. Publ. Math. No. 27 (1965), 55-150. MR0207712 (34:7527)

[8] N. Bourbaki, Éléments de mathématique. Fasc. 37. Groupes et algèbres de Lie. Chapitres 4-6, Actualités Sci. Indust., No. 1337, Hermann, Paris, 1968. MR.0240238 (39:1590)

[9] M. Demazure and A. Grothendieck (eds.), Schémas en groupes, Lecture Notes in Math., vols. 151-153, Springer-Verlag, Berlin-New York, 1970. MR0274458 (43:223a) MR0274459 (43:223b) MR.0274460 (43:223c)

[10] V. Petrov and A. Stavrova, Tits indices over semilocal rings, Transform. Groups 16 (2011), $193-217$. MR.2785501 (2012c:20144)

[11] M. R. Stein, Generators, relations, and coverings of Chevalley groups over commutative rings, Amer. J. Math. 93 (1971), 965-1004. MR0322073 (48:437)

[12] J. Tits, Algebraic and abstract simple groups, Ann. of Math. (2) 80 (1964), 313-329. MR0164968 $(29: 2259)$

[13] L. N. Vaserstein, Normal subgroups of orthogonal groups over commutative rings, Amer. J. Math. 110 (1988), 955-973. MR0961501 (89i:20071)

[14] - Normal subgroups of symplectic groups over rings, K-Theory 2 (1989), 647-673. MR0999398 (90f:20064)

Department of Mathematics and Mechanics, St. Petersburg State University, UniverSitetskaya ul. 28, Stary Petergof, St. Petersburg 198504, Russia

E-mail address: mahalex@gmail.com

FAkUltät Für Mathematik, Universität Duisburg-Essen, Germany

E-mail address: anastasia.stavrova@gmail.com

Received 27/MAY/2010

Translated by A. YU. LUZGAREV 